\title{
A decay result for certain windows generating orthogonal Gabor bases
}

\author{
A.J.E.M. Janssen \\ Philips Research Laboratories, WO-02 \\ 5656 AA, Eindhoven, The Netherlands \\ e-mail: a.j.e.m.janssen@philips.com
}

\begin{abstract}
.
We consider tight Gabor frames $(h, a=1, b=1)$ at critical density with $h$ of the form $Z^{-1}(Z g /|Z g|)$. Here $Z$ is the standard Zak transform and $g$ is an even, real, well-behaved window such that $Z g$ has exactly one zero, at $\left(\frac{1}{2}, \frac{1}{2}\right)$, in $[0,1)^{2}$. We show that $h$ and its Fourier transform have maximal decay as allowed by the Balian-Low theorem. Our result illustrates a theorem of Benedetto, Czaja, Gadziński, and Powell, case $p=q=2$, on sharpness of the Balian-Low theorem.
\end{abstract}

Math Subject Classifications (2000):

42C15, 42C25, 94A12.

Keywords and phrases:

Gabor frame, critical density, Balian-Low theorem. 


\section{Introduction}

We continue the investigations in [5]. Thus we consider Gabor systems $(g, a, b)$ at critical density $a=b=1$, i.e., systems of functions $e^{2 \pi i m \cdot} g(\cdot-n)$, integer $n$ and $m$, with well-behaved windows $g \in L^{2}(\mathbb{R})$. Furthermore, we define the Zak transform $Z$ for $f \in L^{2}(\mathbb{R})$ formally by

$$
(Z f)(t, \nu)=\sum_{k=-\infty}^{\infty} f(t-k) e^{2 \pi i k \nu}, \quad t, \nu \in \mathbb{R},
$$

with inverse $Z^{-1}$ formally given by

$$
\left(Z^{-1} F\right)(t)=\int_{0}^{1} F(t, \nu) d \nu, \quad t \in \mathbb{R}
$$

in which $F \in L_{\text {loc }}^{2}\left(\mathbb{R}^{2}\right)$ satisfies the quasi-periodicity relation

$$
F(t+n, \nu+m)=e^{2 \pi i n \nu} F(t, \nu), \quad t, \nu \in \mathbb{R},
$$

for integer $n, m$. Assuming that $Z g$ has finitely many zeros per unit square, we consider the window $h$ defined by

$$
h=Z^{-1}(Z g /|Z g|),
$$

so that $Z h=Z g /|Z g|$. The associated Gabor system $(h, 1,1)$, consisting of integer time-frequency translates $e^{2 \pi i m} \cdot h(\cdot-n)$ of $h$, is an orthogonal base for $L^{2}(\mathbb{R})$. According to the Balian-Low theorem (see [1] for a comprehensive account) we have that at least one of the numbers

$$
\int_{-\infty}^{\infty} t^{2}|h(t)|^{2} d t, \quad \int_{-\infty}^{\infty} \nu^{2}|H(\nu)|^{2} d \nu
$$

is infinite. Here $H=\mathcal{F} h$ denotes the Fourier transform $\int e^{-2 \pi i \nu t} h(t) d t$, $\nu \in \mathbb{R}$, of $h$.

The operation embodied by formula (1.4) is considered in detail in [5], Sections 3, 4, with particular attention for even, real, rapidly decaying windows $g$ with a single zero, at $\left(\frac{1}{2}, \frac{1}{2}\right)$, in the unit square $[0,1)^{2}$. As examples of windows satisfying these requirements, there are considered in [5] the Gaussian and the hyperbolic secant

$$
g_{1, \gamma}(t)=(2 \gamma)^{1 / 4} e^{-\pi \gamma t^{2}}, \quad g_{2, \gamma}(t)=\left(\frac{\pi \gamma}{2}\right)^{1 / 2} \frac{1}{\cosh \pi \gamma t}, \quad t \in \mathbb{R},
$$


and the two-sided exponential

$$
g_{3, \alpha}(t)=\alpha^{1 / 2} e^{-\alpha|t|}, \quad t \in \mathbb{R},
$$

with $\gamma>0, \alpha>0$. These windows satisfy, in addition,

$$
\frac{\partial Z g}{\partial t}\left(\frac{1}{2}, \frac{1}{2}\right) \neq 0 \neq \frac{\partial Z g}{\partial \nu}\left(\frac{1}{2}, \frac{1}{2}\right)
$$

and in [5], Sec. 4, it is shown that this implies that the associated $h$ is in $L^{1}(\mathbb{R})$.

In this article we show that, for any smooth and rapidly decaying $g$ such that $Z g$ has a convergent Taylor series around $\left(\frac{1}{2}, \frac{1}{2}\right)$ and such that (1.8) holds, the $h$ of (1.4) satisfies

$$
\int_{-\infty}^{\infty} \frac{t^{2}}{(\ln (2+|t|))^{d}}|h(t)|^{2} d t<\infty, \quad \int_{-\infty}^{\infty} \frac{\nu^{2}}{(\ln (2+|\nu|))^{d}}|H(\nu)|^{2} d \nu<\infty
$$

when $d>2$. This result illustrates a theorem of Benedetto, Czaja, Gadziński, and Powell, see [2], on the existence of orthonormal Gabor bases whose generator $h$ satisfies

$$
\int_{-\infty}^{\infty} \frac{|t|^{p}}{(\ln (2+|t|))^{d}}|h(t)|^{2} d t<\infty, \quad \int_{-\infty}^{\infty} \frac{|\nu|^{q}}{(\ln (2+|\nu|))^{d}}|H(\nu)|^{2} d \nu<\infty
$$

where $1<p, q<\infty, p^{-1}+q^{-1}=1$, and $d>2$. An effort as in the present article, with quite different methods, is undertaken in [3] for the case of a basis introduced by Høholdt, Jensen, and Justesen in [4], with $p=\frac{3}{2}, q=3$.

The conditions of well-behavedness on $g$ and $Z g$ can be considerably relaxed. The chosen restrictions are such that the key argument, presented in the next section, admits a convenient work-out. As an illustration that the main result holds more generally, we show that (1.9) also holds for $h=$ $Z^{-1}(Z g /|Z g|)$ with $g=g_{3, \alpha}$ (see (1.7), $g$ not smooth at $\left.t=0\right)$.

\section{Paper outline}

The proof of our main result is based on the detailed analysis conducted in [5], Section 6 for the case that $g=g_{3, \alpha}$, see (1.7). In this case we have

$$
h_{3, \alpha}(t+n)=I_{n}(r(t)), \quad n \in \mathbb{Z}, \quad t \in[0,1),
$$


where $r(t)=\sinh \alpha t / \sinh \alpha(1-t)$, and

$$
I_{n}(r)=\int_{0}^{1} e^{2 \pi i n \nu} \frac{1+r e^{2 \pi i \nu}}{\left|1+r e^{2 \pi i \nu}\right|} d \nu, \quad n \in \mathbb{Z}, \quad r \geq 0 .
$$

In [5], Section 6, B.6, there is shown the inequality

$$
0 \leq(-1)^{n} I_{n}(r) \leq \exp \left(-n \sqrt{2}\left|\frac{1-r}{1+r}\right|\right) \min \left(2, \frac{1+r}{n \sqrt{2 r}}\right)
$$

for $n=0,1, \ldots$ and $r \geq 0$. As a consequence we have that

$$
\int_{n}^{n+1}|h(t)|^{2} d t=O\left(\frac{1}{n^{3}}\right), \quad n=0,1, \ldots
$$

with $h=h_{3, \alpha}$, and it follows that the first integral in (1.9) is finite for $h=h_{3, \alpha}$ when $d>2$. Here the fact that $r^{\prime}\left(\frac{1}{2}\right) \neq 0$ is essential.

Our approach consists now of approximating a general

$$
\frac{(Z g)(t, \cdot)}{|(Z g)(t, \cdot)|} \text { by } \nu \in[0,1) \mapsto \frac{1+r_{g}(t) e^{2 \pi i \nu}}{\mid 1+r_{g}(t) e^{2 \pi i \nu \mid}}
$$

with judiciously chosen $r_{g}(t) \geq 0$ for $t$ near $\frac{1}{2}$. With $h(t+n)$ given as

$$
h(t+n)=\int_{0}^{1} e^{2 \pi i n \nu} \frac{(Z g)(t, \nu)}{|(Z g)(t, \nu)|} d \nu, \quad t \in[0,1), \quad n \in \mathbb{Z},
$$

the choice of $r_{g}(t)$ should be such that we can bound the error $h(t+n)-$ $I_{n}\left(r_{g}(t)\right)$ appropriately. It turns out that we can achieve this goal with a smooth $r_{g}$ satisfying

$$
r_{g}\left(\frac{1}{2}\right)=1, \quad r_{g}^{\prime}\left(\frac{1}{2}\right)>0
$$

the latter being a consequence of the assumption (1.8). Then (2.3) allows us to show that (2.4) holds, and this implies that the first integral in (1.9) is finite.

The finiteness of the second integral in (1.9) is a consequence of Fourier invariance of the class of even, real, well-behaved windows $g$ such that (1.8) holds.

This article is organized as follows. In Section 3 we present preparatory material concerning Zak transforms of even, real, well-behaved windows $g$ 
with unique Zak transform zero in $[0,1)^{2}$ at $\left(\frac{1}{2}, \frac{1}{2}\right)$. In Section 4 we present the proof of the main result in which we consider approximation as in (2.5), bound the error $h(t+n)-I_{n}\left(r_{g}(t)\right)$, and show that the first integral in (1.9) is finite. Next, in Section 5 we show that the second integral in (1.9) is finite. This uses basic Zak transform properties and Fourier invariance of the class of considered $g$ 's. For the window $g=g_{3, \alpha}$, see (1.7), that is not smooth at $t=0$, the situation is somewhat more delicate, and this requires an additional argument, presented in Section 6, to show finiteness of the second integral in (1.9) with $h=h_{3, \alpha}$.

\section{Preparation}

We consider in this section even, real, smooth, rapidly decaying windows $g$ with smooth Zak transforms $Z g$ having a single zero, at $\left(\frac{1}{2}, \frac{1}{2}\right)$, in $[0,1)^{2}$. We shall assume without loss of generality that

$$
(Z g)(0,0)=\sum_{k=-\infty}^{\infty} g(k)>0
$$

Due to the quasi-periodicity relations, see (1.3), we have

$$
(Z g)(t, 1)=(Z g)(t, 0), \quad(Z g)(1, \nu)=e^{2 \pi i \nu}(Z g)(0, \nu) .
$$

Lemma 1. We have for $t, \nu \in[0,1]$

$$
(Z g)\left(t, \frac{1}{2}\right)=A(t), \quad(Z g)\left(\frac{1}{2}, \nu\right)=2 e^{\pi i \nu} B(\nu),
$$

where $A(t)$ is given by

$$
A(t)=\sum_{k=0}^{\infty}(-1)^{k}[g(k+t)-g(k+(1-t))], \quad 0 \leq t \leq 1
$$

and satisfies

$$
A(t)=-A(1-t)>0, \quad 0 \leq t<\frac{1}{2},
$$

while $B(\nu)$ is given by

$$
B(\nu)=\sum_{k=0}^{\infty} g\left(k+\frac{1}{2}\right) \cos 2 \pi\left(k+\frac{1}{2}\right) \nu, \quad 0 \leq \nu \leq 1,
$$

and satisfies

$$
B(\nu)=-B(1-\nu)>0, \quad 0 \leq \nu<\frac{1}{2} .
$$


Proof. This follows easily from the fact that $g$ is real and even, together with the facts that $(Z g)(0,0)>0$ and that $Z g$ vanishes on $[0,1)^{2}$ at $\left(\frac{1}{2}, \frac{1}{2}\right)$ only. Also see the beginning of Section 4 in [5].

Consequences.

(i) $A\left(\frac{1}{2}\right)=B\left(\frac{1}{2}\right)=0$,

(ii) $\frac{\partial Z g}{\partial t}\left(\frac{1}{2}, \frac{1}{2}\right) \leq 0, \frac{1}{i} \frac{\partial Z g}{\partial \nu}\left(\frac{1}{2}, \frac{1}{2}\right) \leq 0$,

(iii) $(Z h)\left(\frac{1}{2}, \nu\right)=(Z g)\left(\frac{1}{2}, \nu\right) /\left|(Z g)\left(\frac{1}{2}, \nu\right)\right|=e^{\pi i \nu} \operatorname{sgn}\left(\frac{1}{2}-\nu\right)$.

\section{Finiteness of the time-domain integral}

In this section we show that the first integral in (1.9) is finite. We assume here that $g$ is even, real, smooth and rapidly decaying, that $Z g$ has a convergent Taylor series around $\left(\frac{1}{2}, \frac{1}{2}\right)$, and that $Z g$ has one zero in $[0,1)^{2}$, at $\left(\frac{1}{2}, \frac{1}{2}\right)$, with

$$
E:=\frac{\partial Z g}{\partial t}\left(\frac{1}{2}, \frac{1}{2}\right) \neq 0 \neq \frac{\partial Z g}{\partial \nu}\left(\frac{1}{2}, \frac{1}{2}\right)=: F
$$

From Consequence (ii) after Lemma 1 we know that $E<0, \frac{1}{i} F<0$.

\subsection{Approximation of $Z g /|Z g|$}

We approximate for $t$ near $\frac{1}{2}$

$$
z_{I}(t, \mu):=\frac{(Z g)\left(t, \frac{1}{2}+\mu\right)}{\left|(Z g)\left(t, \frac{1}{2}+\mu\right)\right|} \text { by } z_{I I}(t, \mu):=\frac{1+r_{g}(t) e^{2 \pi i(1 / 2+\mu)}}{\left|1+r_{g}(t) e^{2 \pi i(1 / 2+\mu)}\right|}
$$

as a function of $\mu$ near $\mu=0$ with judiciously chosen function $r_{g}(t)$. To that end we write for $t$ near $\frac{1}{2}$

$$
(Z g)\left(t, \frac{1}{2}+\mu\right)=b_{0}(t)+b_{1}(t) \mu+b_{2}(t) \mu^{2}+\ldots
$$

with

$$
b_{k}(t)=\frac{1}{k !} \frac{\partial^{k} Z g}{\partial \nu^{k}}\left(t, \frac{1}{2}\right), \quad k=0,1, \ldots .
$$

From $(Z g)\left(t, \frac{1}{2}-\mu\right)=(Z g)^{*}\left(t, \frac{1}{2}+\mu\right)$, we have

$$
i^{k} b_{k}(t) \in \mathbb{R}, \quad k=0,1, \ldots,
$$


and there holds by (4.1) that

$$
b_{0}\left(\frac{1}{2}\right)=(Z g)\left(\frac{1}{2}, \frac{1}{2}\right)=0, \quad b_{0}^{\prime}\left(\frac{1}{2}\right)=E \neq 0 \neq F=b_{1}\left(\frac{1}{2}\right) .
$$

Similarly, we have from $e^{2 \pi i \mu}=1+2 \pi i \mu-2 \pi^{2} \mu^{2}-\ldots$

$$
1+r_{g}(t) e^{2 \pi i(1 / 2+\mu)}=1-r_{g}(t)-2 \pi i r_{g}(t) \mu+2 \pi^{2} r_{g}(t) \mu^{2}+\ldots
$$

For approximation of $z_{I}$ by $z_{I I}$, we take $r_{g}(t)$ such that

$$
\frac{-2 \pi i r_{g}(t)}{1-r_{g}(t)}=\frac{b_{1}(t)}{b_{0}(t)}
$$

for $t$ near $\frac{1}{2}, t \neq \frac{1}{2}$, so that

$$
r_{g}(t)=\frac{i b_{1}(t)}{i b_{1}(t)+2 \pi b_{0}(t)} .
$$

Then $r_{g}(t)$ is well-defined and smooth for $t$ near $\frac{1}{2}$ by smoothness of $b_{0}, b_{1}$ and (4.6), and

$$
r_{g}\left(\frac{1}{2}\right)=1, \quad r_{g}^{\prime}\left(\frac{1}{2}\right)=2 \pi i \frac{b_{0}^{\prime}\left(\frac{1}{2}\right)}{b_{1}\left(\frac{1}{2}\right)}=\frac{2 \pi i E}{F}>0 .
$$

Consequently, $\left(1-r_{g}(t)\right) / b_{0}(t)$ is positive near $t=\frac{1}{2}$, and so we are approximating

$$
z_{I}(t, \mu)=\frac{b_{0}(t)+b_{1}(t) \mu+b_{2}(t) \mu^{2}+\ldots}{\left|b_{0}(t)+b_{1}(t) \mu+b_{2}(t) \mu^{2}+\ldots\right|}
$$

by

$$
z_{I I}(t, \mu)=\frac{b_{0}(t)+b_{1}(t) \mu+\pi i b_{1}(t) \mu^{2}+\ldots}{\left|b_{0}(t)+b_{1}(t) \mu+\pi i b_{1}(t) \mu^{2}+\ldots\right|} .
$$

Lemma 2. We have for $t$ near $\frac{1}{2}$

$$
\begin{gathered}
b_{0}(t)=\left(t-\frac{1}{2}\right) E+O\left(t-\frac{1}{2}\right)^{2}, \\
b_{1}(t)=F+O\left(t-\frac{1}{2}\right), \\
b_{2}(t)-\pi i b_{1}(t)=O\left(t-\frac{1}{2}\right) .
\end{gathered}
$$

Proof. From (4.5) and smoothness of $Z g$ around $\left(\frac{1}{2}, \frac{1}{2}\right)$ we get (4.13) and (4.14). Writing $B\left(\frac{1}{2}+\mu\right)=C(\mu)$ we have from Lemma 1 that $C$ is odd, real and smooth with $C(0)=0 \neq C^{\prime}(0)$. Then

$$
\begin{aligned}
& (Z g)\left(\frac{1}{2}, \frac{1}{2}+\mu\right)=2 i e^{\pi i \mu} C(\mu)= \\
& =2 i\left(1+\pi i \mu-\frac{1}{2} \pi^{2} \mu^{2}-\ldots\right)\left(C^{\prime}(0) \mu+\frac{1}{6} C^{\prime \prime \prime}(0) \mu^{3}+\ldots\right),
\end{aligned}
$$


from which we get

$$
b_{1}\left(\frac{1}{2}\right)=0, \quad b_{1}\left(\frac{1}{2}\right)=2 i C^{\prime}(0), \quad b_{2}\left(\frac{1}{2}\right)=-2 \pi C^{\prime}(0),
$$

and this yields (4.15) by smoothness of $Z g$.

We now write the numerators at the right-hand sides of (4.11) and (4.12) as

$$
\begin{aligned}
w_{I}(t, \mu) & :=b_{0}(t)+b_{1}(t) \mu+b_{2}(t) \mu^{2}+\ldots= \\
& =b_{0}(t)+i d_{I}(t, \mu) \mu+c_{I}(t, \mu) \mu^{2},
\end{aligned}
$$

and

$$
\begin{aligned}
w_{I I}(t, \mu) & :=b_{0}(t)+b_{1}(t) \mu+\pi i b_{1}(t) \mu^{2}+\ldots= \\
& =b_{0}(t)+i d_{I I}(t, \mu) \mu+c_{I I}(t, \mu) \mu^{2}
\end{aligned}
$$

respectively. Here

$$
\begin{aligned}
& i d_{I}(t, \mu)=b_{1}(t)+b_{3}(t) \mu^{2}+\ldots, \quad i d_{I I}(t, \mu)=b_{1}(t)-\frac{2}{3} \pi^{2} b_{1}(t) \mu^{2}+\ldots, \\
& c_{I}(t, \mu)=b_{2}(t)+b_{4}(t) \mu^{2}+\ldots, \quad c_{I I}(t, \mu)=\pi i b_{1}(t)-\frac{1}{3} \pi^{2} i b_{1}(t) \mu^{2}+\ldots,
\end{aligned}
$$

respectively. We observe that $b_{0}, d_{I, I I}$ and $c_{I, I I}$ are real. Furthermore, by Lemma 2,

$$
\left|b_{0}(t)\right| \geq \frac{1}{2}|E|\left|t-\frac{1}{2}\right|, \quad\left|d_{I, I I}(t, \mu)\right| \geq \frac{1}{2}|F|
$$

and

$$
\begin{gathered}
b_{0}(t)=O\left(t-\frac{1}{2}\right) ; \quad d_{I, I I}(t, \mu), \quad c_{I, I I}(t, \mu)=O(1), \\
d_{I}(t, \mu)-d_{I I}(t, \mu)=O\left(\mu^{2}\right), \quad c_{I}(t, \mu)-c_{I I}(t, \mu)=O\left(t-\frac{1}{2}\right)+O\left(\mu^{2}\right) .
\end{gathered}
$$

In (4.22)-(4.24), the inequalities and $O$ 's hold in a set $\left(\frac{1}{2}-\delta_{1}, \frac{1}{2}+\delta_{1}\right) \times\left(-\delta_{2}, \delta_{2}\right)$ of $(t, \mu)$ 's with $\delta_{1}>0, \delta_{2}>0$.

In the statement and proof of the next lemma, we omit the variable $t$ in $b_{0}$ and the variables $t, \mu$ in $d_{I, I I}, c_{I, I I}, w_{I, I I}$ and $z_{I, I I}$.

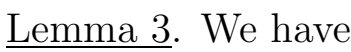
(i) $\left|z_{I}-z_{I I}\right|=O\left(\mu^{2}\right)$,
(ii) $\left|z_{I}^{\prime}-z_{I I}^{\prime}\right|=O(\mu)$, 
where the ' denotes differentiation with respect to $\mu$, and the $O$ 's hold for $|\mu| \leq \frac{1}{2}$ and $t$ near $\frac{1}{2}, t \neq \frac{1}{2}$.

Proof. By smoothness of $z_{I, I I}$ in a set $\left(\frac{1}{2}-\delta_{1}, \frac{1}{2}+\delta_{1}\right) \times\left[-\frac{1}{2}, \frac{1}{2}\right) \backslash\left\{\left(\frac{1}{2}, 0\right)\right\}$, it is sufficient to establish (i) and (ii) for $(t, \mu)$ in a set $\left(\left(\frac{1}{2}-\delta_{1}, \frac{1}{2}+\delta_{1}\right) \backslash\left\{\frac{1}{2}\right\}\right) \times$ $\left(-\delta_{2}, \delta_{2}\right)$, and so we restrict $(t, \mu)$ to such a set.

(i) With $w_{I, I I}$ given by (4.18)-(4.19), we have

$$
z_{I}-z_{I I}=\frac{w_{I}}{\left|w_{I}\right|}-\frac{w_{I I}}{\left|w_{I I}\right|}=\frac{w_{I}-w_{I I}}{\left|w_{I}\right|}+w_{I I}\left(\frac{1}{\left|w_{I}\right|}-\frac{1}{\left|w_{I I}\right|}\right) .
$$

We shall bound $\left|w_{I, I I}\right|$ from below and estimate $w_{I}-w_{I I}$. By the second item in (4.22) there holds

$$
\left|w_{I, I I}\right| \geq \frac{1}{2}|F||\mu|, \quad 0<\left|t-\frac{1}{2}\right|<\delta_{1}, \quad|\mu|<\delta_{2} .
$$

Also, from the first item in (4.22) and (4.23), there are $G>0, H>0$, such that

$$
\begin{aligned}
\left|w_{I, I I}\right|=\left|b_{0}+c_{I, I I} \mu^{2}+i d_{I, I I} \mu\right| \geq G\left|t-\frac{1}{2}\right|, & \\
0 & <\left|t-\frac{1}{2}\right|<\delta_{1}, \quad|\mu|<H\left|t-\frac{1}{2}\right|^{1 / 2} .
\end{aligned}
$$

We next have from (4.24)

$$
\begin{aligned}
w_{I I} & =w_{I}+i\left(d_{I I}-d_{I}\right) \mu+\left(c_{I I}-c_{I}\right) \mu^{2}= \\
& =w_{I}+O\left(\mu^{3}\right)+O\left(\mu^{2}\left(t-\frac{1}{2}\right)\right)= \\
& =w_{I}+O\left(\mu^{2}\right)\left(O(\mu)+O\left(t-\frac{1}{2}\right)\right) .
\end{aligned}
$$

From (4.26)-(4.27) we have

$$
\begin{aligned}
& \frac{O(\mu)+O\left(t-\frac{1}{2}\right)}{\left|w_{I}\right|}= \\
& =O(1)+\left\{\begin{array}{l}
O(1) \quad, \quad 0<\left|t-\frac{1}{2}\right|<\delta_{1}, \quad|\mu|<H\left|t-\frac{1}{2}\right|^{1 / 2} \\
O\left(\frac{t-\frac{1}{2}}{\mu}\right), \quad 0<\left|t-\frac{1}{2}\right|<\delta_{1}, \quad|\mu| \geq H\left|t-\frac{1}{2}\right|^{1 / 2}=
\end{array}\right. \\
& =O(1), \quad 0<\left|t-\frac{1}{2}\right|<\delta_{1}, \quad|\mu|<\delta_{2},
\end{aligned}
$$

and therefore

$$
w_{I I}=w_{I}\left(1+O\left(\mu^{2}\right)\right), \quad 0<\left|t-\frac{1}{2}\right|<\delta_{1}, \quad|\mu|<\delta_{2} .
$$


By (4.30) we then have

$$
\frac{w_{I}-w_{I I}}{\left|w_{I}\right|}=O\left(\mu^{2}\right), \quad \frac{1}{\left|w_{I}\right|}-\frac{1}{\left|w_{I I}\right|}=\frac{1}{\left|w_{I}\right|} O\left(\mu^{2}\right),
$$

and since $w_{I I} / w_{I}=O(1)$, we see from (4.25) that $z_{I}-z_{I I}=O\left(\mu^{2}\right)$, as required.

(ii) There holds

$$
z_{I, I I}^{\prime}=\left(\frac{w_{I, I I}}{\left|w_{I, I I}\right|}\right)^{\prime}=\frac{w_{I, I I}^{\prime}}{\left|w_{I, I I}\right|}-z_{I, I I} \frac{\left|w_{I, I I}\right|^{\prime}}{\left|w_{I, I I}\right|} .
$$

Therefore

$$
z_{I}^{\prime}-z_{I I}^{\prime}=\left(\frac{w_{I}^{\prime}}{\left|w_{I}\right|}-\frac{w_{I I}^{\prime}}{\left|w_{I I}\right|}\right)-\left(z_{I} \frac{\left|w_{I}\right|^{\prime}}{\left|w_{I}\right|}-z_{I I} \frac{\left|w_{I I}\right|^{\prime}}{\left|w_{I I}\right|}\right) .
$$

For the first term at the right-hand side of (4.33) we have

$$
\frac{w_{I}^{\prime}}{\left|w_{I}\right|}-\frac{w_{I I}^{\prime}}{\left|w_{I I}\right|}=\frac{w_{I}^{\prime}-w_{I I}^{\prime}}{\left|w_{I}\right|}+w_{I I}^{\prime}\left(\frac{1}{\left|w_{I}\right|}-\frac{1}{\left|w_{I I}\right|}\right) \text {. }
$$

There holds by (4.18)-(4.19) and (4.23) that

$$
\begin{aligned}
w_{I, I I}^{\prime} & =i d_{I, I I}+i d_{I, I I}^{\prime} \mu+2 c_{I, I I} \mu+c_{I, I I}^{\prime} \mu^{2}= \\
& =b_{1}(t)+2 \pi i b_{1}(t) \mu+O\left(\mu^{2}\right)+O\left(\mu\left(t-\frac{1}{2}\right)\right),
\end{aligned}
$$

where also Lemma 2 has been used. In particular, $w_{I I}^{\prime}=O(1)$, and it follows from the second item in (4.31) and from (4.26) that

$$
w_{I I}^{\prime}\left(\frac{1}{\left|w_{I}\right|}-\frac{1}{\left|w_{I I}\right|}\right)=O(\mu) .
$$

Furthermore, by (4.29) and (4.35),

$$
\frac{w_{I}^{\prime}-w_{I I}^{\prime}}{\left|w_{I}\right|}=O(\mu) \frac{O(\mu)+O\left(t-\frac{1}{2}\right)}{\left|w_{I}\right|}=O(\mu) .
$$

We thus conclude from (4.34), (4.36)-(4.37) that

$$
\frac{w_{I}^{\prime}}{\left|w_{I}\right|}-\frac{w_{I I}^{\prime}}{\left|w_{I I}\right|}=O(\mu), \quad 0<\left|t-\frac{1}{2}\right|<\delta_{1}, \quad|\mu|<\delta_{2} .
$$

We proceed by estimating the second at the right-hand side of (4.33), for which we have

$$
z_{I} \frac{\left|w_{I}\right|^{\prime}}{\left|w_{I}\right|}-z_{I I} \frac{\left|w_{I I}\right|^{\prime}}{\left|w_{I I}\right|}=z_{I}\left(\frac{\left|w_{I}\right|^{\prime}}{\left|w_{I}\right|}-\frac{\left|w_{I I}\right|^{\prime}}{\left|w_{I I}\right|}\right)+\left(z_{I}-z_{I I}\right) \frac{\left|w_{I I}\right|^{\prime}}{\left|w_{I I}\right|} .
$$


Since $\left|w_{I I}\right|^{\prime} \leq\left|w_{I I}^{\prime}\right|=O(1)$, we see from (i) $z_{I}-z_{I I}=O\left(\mu^{2}\right)$ and (4.26) that the second term at the right-hand side of (4.39) satisfies

$$
\left(z_{I}-z_{I I}\right) \frac{\left|w_{I I}\right|^{\prime}}{\left|w_{I I}\right|}=O(\mu) .
$$

For the first term on the right-hand side of (4.39) we have

$$
\frac{\left|w_{I}\right|^{\prime}}{\left|w_{I}\right|}-\frac{\left|w_{I I}\right|^{\prime}}{\left|w_{I I}\right|}=\frac{\left|w_{I}\right|^{\prime}-\left|w_{I I}\right|^{\prime}}{\left|w_{I}\right|}+\left|w_{I I}\right|^{\prime}\left(\frac{1}{\left|w_{I}\right|}-\frac{1}{\left|w_{I I}\right|}\right) .
$$

The second term at the right-hand side of (4.41) satisfies

$$
\left|w_{I I}\right|^{\prime}\left(\frac{1}{\left|w_{I}\right|}-\frac{1}{\left|w_{I I}\right|}\right)=O(\mu)
$$

as before (compare (4.36)). For the first term at the right-hand of (4.41) we have to consider $\left|w_{I, I I}\right|^{\prime}$. There holds

$$
\left|w_{I, I I}\right|^{\prime}=\left[\left(\left(b_{0}+c_{I, I I} \mu^{2}\right)^{2}+d_{I, I I}^{2} \mu^{2}\right)^{1 / 2}\right]^{\prime}=\frac{Q_{I, I I}}{\left|w_{I, I I}\right|},
$$

where, see (4.18)-(4.21),

$$
\begin{aligned}
Q_{I, I I} & =\left(b_{0}+c_{I, I I} \mu^{2}\right)\left(c_{I, I I}^{\prime} \mu^{2}+2 c_{I, I I} \mu\right)+d_{I, I I} d_{I, I I}^{\prime} \mu^{2}+d_{I, I I}^{2} \mu= \\
& =2 b_{0} c_{I, I I} \mu+\left|b_{1}(t)\right|^{2} \mu+O\left(\mu^{3}\right)=O(\mu)
\end{aligned}
$$

By (4.23)-(4.24) we have

$$
Q_{I}-Q_{I I}=2 b_{0}\left(c_{I}-c_{I I}\right) \mu+O\left(\mu^{3}\right)=O\left(\left(t-\frac{1}{2}\right)^{2} \mu\right)+O\left(\mu^{3}\right) .
$$

We use (4.44)-(4.45) by writing

$$
\left|w_{I}\right|^{\prime}-\left|w_{I I}\right|^{\prime}=\frac{Q_{I}}{\left|w_{I}\right|}-\frac{Q_{I I}}{\left|w_{I I}\right|}=\frac{Q_{I}-Q_{I I}}{\left|w_{I}\right|}+Q_{I I}\left(\frac{1}{\left|w_{I}\right|}-\frac{1}{\left|w_{I I}\right|}\right) .
$$

By (4.44), (4.26) and the second item in (4.31), we have that

$$
Q_{I I}\left(\frac{1}{\left|w_{I}\right|}-\frac{1}{\left|w_{I I}\right|}\right)=O\left(\mu^{2}\right)
$$

Furthermore, by (4.45) and (4.26),

$$
\frac{Q_{I}-Q_{I I}}{\left|w_{I}\right|}=O\left(\mu^{2}\right)+\frac{O\left(\left(t-\frac{1}{2}\right)^{2} \mu\right)}{\left|w_{I}\right|} .
$$


Now, by (4.26) and (4.27),

$$
\begin{aligned}
& \frac{\left(t-\frac{1}{2}\right)^{2} \mu}{\left|w_{I}\right|}= \\
& =\left\{\begin{array}{lll}
O\left(\left(t-\frac{1}{2}\right) \mu\right) \quad, & 0<\left|t-\frac{1}{2}\right|<\delta_{1}, & |\mu|<H\left|t-\frac{1}{2}\right|^{1 / 2}, \\
O\left(\left(t-\frac{1}{2}\right)^{2}\right)=O\left(\left(t-\frac{1}{2}\right) \mu^{2}\right), & 0<\left|t-\frac{1}{2}\right|<\delta_{1}, & |\mu| \geq H\left|t-\frac{1}{2}\right|^{1 / 2} .
\end{array}\right.
\end{aligned}
$$

Therefore

$$
\frac{Q_{I}-Q_{I I}}{\left|w_{I}\right|}=O\left(\mu^{2}\right)+O\left(\left(t-\frac{1}{2}\right) \mu\right), \quad 0<\left|t-\frac{1}{2}\right|<\delta_{1}, \quad|\mu|<\delta_{2} .
$$

Thus we have from (4.46), (4.47) and (4.50) that

$$
\left|w_{I}\right|^{\prime}-\left|w_{I I}\right|^{\prime}=O\left(\mu^{2}\right)+O\left(\left(t-\frac{1}{2}\right) \mu\right), \quad 0<\left|t-\frac{1}{2}\right|<\delta_{1}, \quad|\mu|<\delta_{2} .
$$

This gives

$$
\frac{\left|w_{I}\right|^{\prime}-\left|w_{I I}\right|^{\prime}}{\left|w_{I}\right|}=O(\mu) \frac{O(\mu)+O\left(t-\frac{1}{2}\right)}{\left|w_{I}\right|}=O(\mu),
$$

see (4.29). Then from (4.41), (4.42) and (4.52) we get

$$
\frac{\left|w_{I}\right|^{\prime}}{\left|w_{I}\right|}-\frac{\left|w_{I I}\right|^{\prime}}{\left|w_{I I}\right|}=O(\mu),
$$

and from (4.40), (4.53) and $\left|z_{I}\right|=1$ we get, see (4.39),

$$
z_{I} \frac{\left|w_{I}\right|^{\prime}}{\left|w_{I}\right|}-z_{I I} \frac{\left|w_{I I}\right|^{\prime}}{\left|w_{I I}\right|}=O(\mu)
$$

so that, finally, from (4.33), (4.38) and (4.54), we obtain

$$
\left|z_{I}^{\prime}-z_{I I}^{\prime}\right|=O(\mu), \quad 0<\left|t-\frac{1}{2}\right|<\delta_{1}, \quad 0<|\mu|<\delta_{2}
$$

as required. 


\subsection{Estimating $h(t+n)$ and completion of the proof}

We consider for $n=0,1, \ldots, t \in[0,1), t$ close to $\frac{1}{2}$,

$$
\begin{aligned}
h(t+n)-I_{n}\left(r_{g}(t)\right) & =\int_{0}^{1} e^{2 \pi i n \nu}\left(\frac{(Z g)(t, \nu)}{|(Z g)(t, \nu)|}-\frac{1+r_{g}(t) e^{2 \pi i \nu}}{\left|1+r_{g}(t) e^{2 \pi i \nu}\right|}\right) d \nu= \\
& =(-1)^{n} \int_{-1 / 2}^{1 / 2} e^{2 \pi i n \mu}\left(z_{1}(t, \mu)-z_{2}(t, \mu)\right) d \mu
\end{aligned}
$$

By partial integration and periodicity, we get

$$
h(t+n)-I_{n}\left(r_{g}(t)\right)=\frac{-(-1)^{n}}{2 \pi i n} \int_{-1 / 2}^{1 / 2} e^{2 \pi i n \mu} \frac{\partial}{\partial \mu}\left(z_{1}(t, \mu)-z_{2}(t, \mu)\right) d \mu .
$$

By Parseval's theorem, we subsequently estimate

$$
\begin{aligned}
& \sum_{n=0}^{\infty} n^{2}\left|h(t+n)-I_{n}\left(r_{g}(t)\right)\right|^{2} \leq \\
& \leq \frac{1}{4 \pi^{2}} \sum_{n=-\infty}^{\infty}\left|\int_{-1 / 2}^{1 / 2} e^{2 \pi i n \mu} \frac{\partial}{\partial \mu}\left(z_{1}(t, \mu)-z_{2}(t, \mu)\right) d \mu\right|^{2}= \\
& =\frac{1}{4 \pi^{2}}\left\|\frac{\partial}{\partial \mu}\left(z_{1}(t, \cdot)-z_{2}(t, \cdot)\right)\right\|_{L^{2}([-1 / 2,1 / 2])}^{2} \cdot
\end{aligned}
$$

The far right-hand side of (4.58) is bounded in $t$ close to $\frac{1}{2}$ by Lemma 4 (ii), say for $t \in\left[\frac{1}{2}-\delta_{1}, \frac{1}{2}+\delta_{1}\right]$. By smoothness of $Z g /|Z g|$ in $\left(\left[0, \frac{1}{2}-\delta_{1}\right) \cup\left(\frac{1}{2}+\right.\right.$ $\left.\left.\delta_{1}, 1\right)\right) \times[0,1)$ and $(2.6)$ we have that

$$
\sum_{n=0}^{\infty}\left(\int_{0}^{1 / 2-\delta_{1}}+\int_{1 / 2+\delta_{1}}^{1}\right)\left(1+(t+n)^{2}\right)|h(t+n)|^{2} d t<\infty .
$$

Let $d>2$. For proving that

$$
\int_{0}^{\infty} \frac{1+t^{2}}{(\ln (2+t))^{d}}|h(t)|^{2} d t<\infty
$$


it is therefore sufficient to show that

$$
\sum_{n=0}^{\infty} \int_{1 / 2-\delta_{1}}^{1 / 2+\delta_{1}} \frac{1+(t+n)^{2}}{(\ln (2+(t+n)))^{d}} I_{n}^{2}\left(r_{g}(t)\right) d t<\infty .
$$

To show (4.61), we apply (2.3) where we note that $r_{g}(t)$ is close to 1 and $r_{g}^{\prime}(t)$ is strictly positive on $\left[\frac{1}{2}-\delta_{1}, \frac{1}{2}+\delta_{1}\right]$ by (4.10) and smoothness of $r_{g}$. Consequently,

$$
\int_{1 / 2-\delta_{1}}^{1 / 2+\delta_{1}} I_{n}^{2}\left(r_{g}(t)\right) d t=O\left(\frac{1}{n^{3}}\right), \quad n=1,2, \ldots,
$$

and this implies (4.61). Therefore (4.60) holds, and since $h$ is even, we see that the first integral in (1.9) is finite.

Comment. Lemma 4 gives more than enough to complete the proof of finiteness of the time-domain integral; in fact, boundedness or integrability of the far right-hand side of (4.58) as a function of $t$ near $\frac{1}{2}$ is enough. There is, however, the following bonus. In [5], it has been observed how similar the graphs of two windows $h=Z^{-1}(Z g /|Z g|)$ may look, even though the windows $g$ are quite different, when time scalings are set appropriately. Lemma 3, (4.10) and Consequence (iii) at the end of Section 3 come very close to explaining this observation for the type of windows $g$ we have here. Indeed, for these windows, the corresponding $h$ 's are well located in small intervals around the half-integers $n+\frac{1}{2}$. By (2.6) we can therefore limit attention mainly to $(Z h)(t, \cdot)$ with $t$ near $\frac{1}{2}$. By the above mentioned Consequence (iii), all $(Z h)\left(\frac{1}{2}, \cdot\right)$ are the same, viz.

$$
e^{\pi i \nu} \operatorname{sgn}\left(\frac{1}{2}-\nu\right)=\left.\frac{1+r e^{2 \pi i \nu}}{\left|1+r e^{2 \pi i \nu}\right|}\right|_{r=1}, \quad \nu \in[0,1) .
$$

By smoothness of $Z g$, we have that $(Z g)(t, \cdot)$ is close to this common function in a $\nu$-set $\left[0, \frac{1}{2}-\varepsilon\right) \cup\left(\frac{1}{2}+\varepsilon, 1\right)$ when $t$ is near $\frac{1}{2}$. The remaining $\nu$-set $\left[\frac{1}{2}-\varepsilon, \frac{1}{2}+\varepsilon\right]$, that yields the main contribution to $h$ near the half-integers, can be dealt with using Lemma 3 and (4.10). According to (4.10), the various $h$ 's can be sorted according to the value of $\partial_{t} Z g / \partial_{\nu} Z g$ at $(t, \nu)=\left(\frac{1}{2}, \frac{1}{2}\right)$. Thus, $h$ 's for which this value is the same have equal values of $r_{g}^{\prime}\left(\frac{1}{2}\right)$, whence the approximating $z_{2}$ 's agree to a considerable extent for $t$ near $\frac{1}{2}$.

As an example, consider the standard Gaussian $g(t)=2^{1 / 4} \exp \left(-\pi t^{2}\right)$ and the two-sided exponential $g_{3, \alpha}(t)=\alpha^{1 / 2} \exp (-\alpha|t|)$ as in the beginning 
of Section 2 in [5]. Now there holds

$$
r_{g}^{\prime}\left(\frac{1}{2}\right)=2 \pi, \quad r_{g_{3, \alpha}}^{\prime}\left(\frac{1}{2}\right)=2 \alpha \operatorname{coth}(\alpha / 2) .
$$

Solving $2 \pi=2 \alpha \operatorname{coth}(\alpha / 2)$ yields $\alpha=2.7717$, and this is not far from the value $\alpha=\sqrt{2 \pi}=2.5066$ that was chosen in [5], Figure 1 (for the latter choice we have $\left.\int t^{2}|g(t)|^{2} d t=\int t^{2}\left|g_{3, \alpha}(t)\right|^{2} d t\right)$.

\section{$5 \quad$ Finiteness of the frequency-domain integral}

We shall now show that the second integral in (1.9) is finite. To that end we note that, with $G=\mathcal{F} g$, we have

$$
(Z G)(t, \nu)=e^{2 \pi i \nu t}(Z g)(-\nu, t)=e^{-2 \pi i(1-\nu) t}(Z g)(1-\nu, t), \quad t, \nu \in \mathbb{R} .
$$

Hence, smoothness of $Z G$ is implied by smoothness of $Z g$. Furthermore,

$$
\frac{\partial Z G}{\partial t}\left(\frac{1}{2}, \frac{1}{2}\right)=-i \frac{\partial Z g}{\partial \nu}\left(\frac{1}{2}, \frac{1}{2}\right), \quad \frac{\partial Z G}{\partial \nu}\left(\frac{1}{2}, \frac{1}{2}\right)=i \frac{\partial Z g}{\partial t}\left(\frac{1}{2}, \frac{1}{2}\right) .
$$

Hence, by (1.8) we have that $\frac{\partial Z G}{\partial t}$ and $\frac{\partial Z G}{\partial \nu}$ do not vanish at $\left(\frac{1}{2}, \frac{1}{2}\right)$. Finally, from (5.1), holding with $H=\mathcal{F} h$ instead of $G=\mathcal{F} g$ as well, we have that

$$
H=Z^{-1}(Z G /|Z G|)
$$

We conclude that we can apply the result proved in the previous section with $H$ instead of $h$, and so the second integral in (1.9) is finite.

\section{Proof of the main result for $g=g_{3, \alpha}$}

We have noted in the beginning of Section 2 that the first integral in (1.9) is finite when $h=Z^{-1}(Z g /|Z g|)$ and $g=g_{3, \alpha}$, see (1.7). To show finiteness of the second integral, the argument used in Section 5 cannot be applied since $g_{3, \alpha}$ has a discontinuous derivative at $t=0$, causing $\partial Z g / \partial t$ to be discontinuous at all $t=n \in \mathbb{Z}$. We shall argue, therefore, more carefully. In [5], Sections 5, 6, the Zak transforms of $g_{3, \alpha}$ and $h_{3, \alpha}$ have been calculated. Writing $H_{3, \alpha}=\mathcal{F} h_{3, \alpha}$, we thus have

$$
H_{3, \alpha}(\nu)=\int_{0}^{1} e^{-2 \pi i \nu t}\left(Z h_{3, \alpha}\right)(t, \nu) d t=\int_{0}^{1} e^{-2 \pi i m t} e^{-2 \pi i \sigma t} \frac{1+r(t) e^{2 \pi i \sigma}}{\left|1+r(t) e^{2 \pi i \sigma}\right|} d t
$$


with $r(t)=\sinh \alpha t / \sinh \alpha(1-t)$ and where we have set $\nu=m+\sigma, m \in \mathbb{Z}$, $\sigma \in[0,1)$. Denote $s(t)=\sinh \alpha t$, and consider

$$
\begin{aligned}
W(t, \sigma) & =e^{-2 \pi i \sigma t} \frac{1+r(t) e^{2 \pi i \sigma}}{\left|1+r(t) e^{2 \pi i \sigma}\right|}= \\
& =\frac{s(1-t) e^{-2 \pi i \sigma t}+s(t) e^{2 \pi i \sigma(1-t)}}{\mid s(1-t) e^{-2 \pi i \sigma t}+s(t) e^{2 \pi i \sigma(1-t) \mid}}, \quad(t, \sigma) \in[0,1)^{2} .
\end{aligned}
$$

The function

$$
N(t, \sigma)=s(1-t) e^{-2 \pi i \sigma t}+s(t) e^{2 \pi i \sigma(1-t)}, \quad(t, \sigma) \in[0,1)^{2},
$$

is smooth, satisfies $N(0, \sigma)=N(1, \sigma)$, and $\partial N / \partial t \neq 0 \neq \partial N / \partial \sigma$ at $\left(\frac{1}{2}, \frac{1}{2}\right)$. Hence, all what has been done in Section 4 applies here, except that we have to check whether $W(\cdot, \sigma)$ is smooth enough at $t=0,1$ as a 1 -periodic function of $t$. Compare (6.1) and (2.6).

Writing $W(t, \sigma)=\exp (i \psi(t))$ with

$$
\psi(t)=\operatorname{Im}\left[\ln \left(s(1-t) e^{-2 \pi i \sigma t}+s(t) e^{2 \pi i \sigma(1-t)}\right)\right],
$$

a smooth function when $\sigma \neq \frac{1}{2}$, we compute

$$
\frac{\partial W}{\partial t}(t, \sigma)=i \operatorname{Im}\left[\frac{\left(s(1-t) e^{-2 \pi i \sigma t}+s(t) e^{2 \pi i \sigma(1-t)}\right)^{\prime}}{s(1-t) e^{-2 \pi i \sigma t}+s(t) e^{2 \pi i \sigma(1-t)}}\right] W(t, \sigma)
$$

where the ' now indicates derivative with respect to $t$. At $t=0+, 1-$ we have

$$
s(1-t) e^{-2 \pi i \sigma t}+s(t) e^{2 \pi i \sigma(1-t)}=s(1)
$$

while

$$
\begin{aligned}
& \left(s(1-t) e^{-2 \pi i \sigma t}+s(t) e^{2 \pi i \sigma(1-t)}\right)^{\prime}= \\
& = \begin{cases}-s^{\prime}(1)+s^{\prime}(0) \cos 2 \pi \sigma-2 \pi i \sigma s(1)+i s^{\prime}(0) \sin 2 \pi \sigma, & t=0+ \\
+s^{\prime}(1)-s^{\prime}(0) \cos 2 \pi \sigma-2 \pi i \sigma s(1)+i s^{\prime}(0) \sin 2 \pi \sigma, & t=1-\end{cases}
\end{aligned}
$$

Hence, $W(\cdot, \sigma)$ is continuously differentiable at $t=0,1$ since (6.5) requires only imaginary parts of (6.7). By a standard argument using partial integration, also see Subsection 4.2, it follows that we do not need to bother about the boundary points $t=0,1$ of the integration interval in (6.1). 


\section{Acknowledgements}

The author is grateful to the referee who pointed out serious shortcomings in an earlier version. This has led to better results and better presentation in the final version of the paper.

\section{References}

[1] Benedetto, J.J., Heil, C. and Walnut, D. (1995). Differentiation and the Balian-Low theorem, J. Four. Anal. Appl., 1 (4), 355-402.

[2] Benedetto, J.J., Czaja, W., Gadziński, P. and Powell, A.M. (2003). The Balian-Low theorem and regularity of Gabor systems, J. Geom. Anal., 13 (2), 239-254.

[3] Benedetto, J.J., Czaja, W. and Powell, A.M. (2006). Optimality in the Balian-Low theorem and a basis of Høholdt, Jensen, and Justesen, SIAM J. Math. Anal., 38 (1), 333-345.

[4] Jensen, H., Høholdt, T. and Justesen, J. (1988). Double series representation of bounded signals, IEEE Trans. Inform. Theory, 34 (4), 613-624.

[5] Janssen, A.J.E.M. (2003). On generating tight Gabor frames at critical density, J. Four. Anal. Appl., 9 (2), 175-214. 